\title{
Enabling Computational Technologies for Subsurface Simulations
}

\author{
R. D. Falgout, C. Baldwin, W. Bosl, R. Hornung,
} D. Shumaker, S. Smith, C. Woodward, A. F. B. Tompson

February 22, 1999

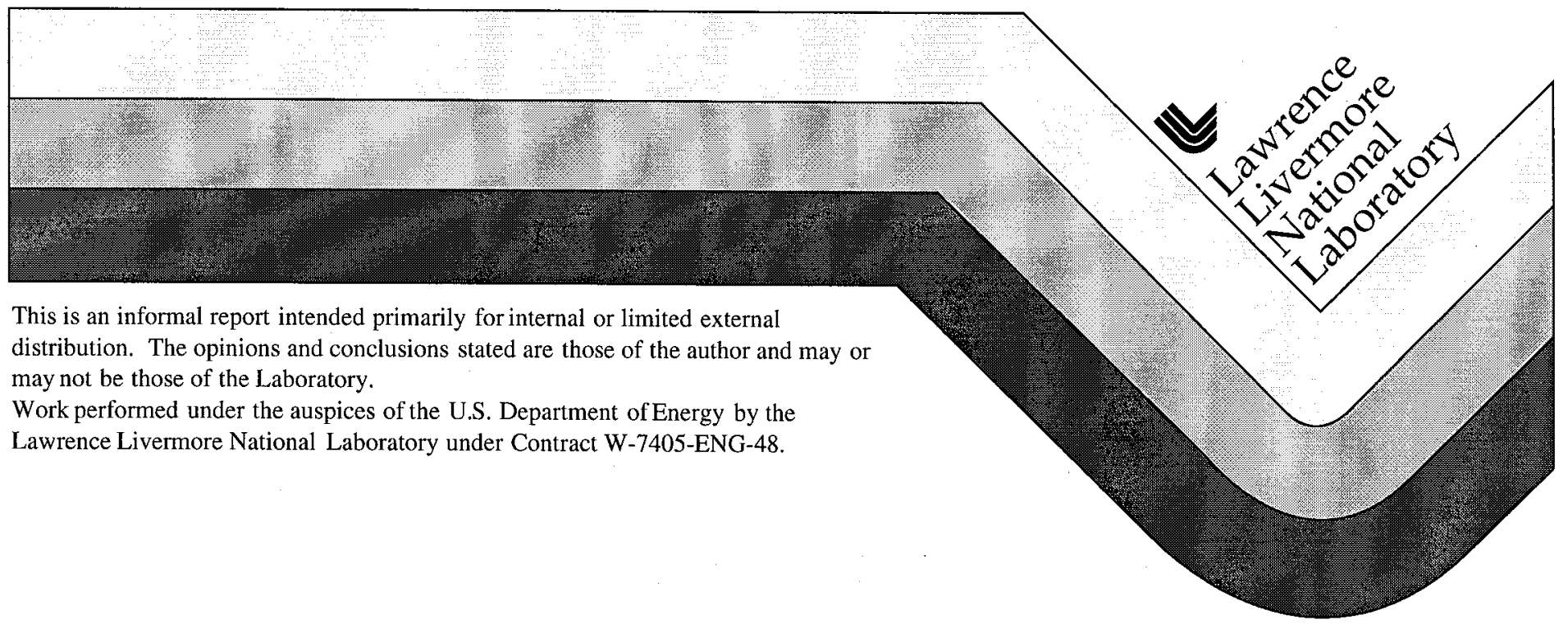




\section{DISCLAIMER}

This document was prepared as an account of work sponsored by an agency of the United States Government. Neither the United States Government nor the University of California nor any of their employees, makes any warranty, express or implied, or assumes any legal liability or responsibility for the accuracy, completeness, or usefulness of any information, apparatus, product, or process disclosed, or represents that its use would not infringe privately owned rights. Reference herein to any specific commercial product, process, or service by trade name, trademark, manufacturer, or otherwise, does not necessarily constitute or imply its endorsement, recommendation, or favoring by the United States Government or the University of California. The views and opinions of authors expressed herein do not necessarily state or reflect those of the United States Government or the University of California, and shall not be used for advertising or product endorsement purposes.

This report has been reproduced directly from the best available copy.

Available to DOE and DOE contractors from the Office of Scientific and Technical Information P.O. Box 62, Oak Ridge, TN 37831

Prices available from (615) 576-8401, FTS 626-8401

Available to the public from the National Technical Information Service

U.S. Department of Commerce 5285 Port Royal Rd. Springfield, VA 22161 
An LDRD Final Report

\title{
Enabling Computational Technologies for Subsurface Simulations
}

\author{
Project 97-ERD-035 \\ Robert D. FALGOUT (Principal Investigator) \\ Center for Applied Scientific Computing \\ C. Baldwin, W. Bosl, R. Hornung, D. Shumaker, S. Smith, C. Woodward \\ Center for Applied Scientific Computing \\ ANDREW F. B. TOMPSON \\ Earth Sciences Division (Environmental Programs)
}

\section{Summary}

We collaborated with Environmental Programs to develop and apply advanced computational methodologies for simulating multiphase flow through heterogeneous porous media. The primary focus was on developing a fast accurate advection scheme using a new temporal subcycling technique and on the scalable and efficient solution of the nonlinear Richards' equation used to model two-phase (variably saturated) flow. The resulting algorithms can be orders-of-magnitude faster than existing methods. Our computational technologies were applied to the simulation of subsurface fluid flow and chemical transport in the context of two important applications: water resource management and groundwater remediation.

\section{Problem Description and Project Objectives}

The numerical simulation of fluid flow and chemical transport through the subsurface is a fundamental computational challenge arising in several important applications, including water resource management and groundwater remediation. Such fluid flows are characterized by three-dimensional multiphase flow in heterogeneous porous media, coupled to multiple reacting chemical species. In water resource management, simulations have traditionally been used for the purposes of planning extraction well placement and design and understanding integrated recharge behavior. Although these are still important problems, more immediate and emerging problems in this area center on water quality issues such as those related to recharge and reuse of recycled water (e.g., treated wastewater), impacts of historical agricultural practices (e.g., fertilizers), increased use and exploitation of groundwater resources, and salt water intrusion. In groundwater remediation, simulations are typically used to determine the basic flow and chemical migration patterns at contaminated sites, analyze, develop, and optimize remediation strategies for the removal of contaminants, demonstrate compliance with regulatory cleanup standards, and evaluate contaminant travel times and attenuation rates for use in environmental risk analyses [11].

It has been shown that fine-scale heterogeneity has a dramatic impact on fluid flow and chemical transport (see, e.g., $[1,2,16]$ ). For example, Figure 1 shows a snapshot in time of a contaminant migrating through a homogeneous and a heterogeneous subsurface medium. Here, the heterogeneities have a visible effect on the dispersion and transport speed of the contaminant, due to the presence 

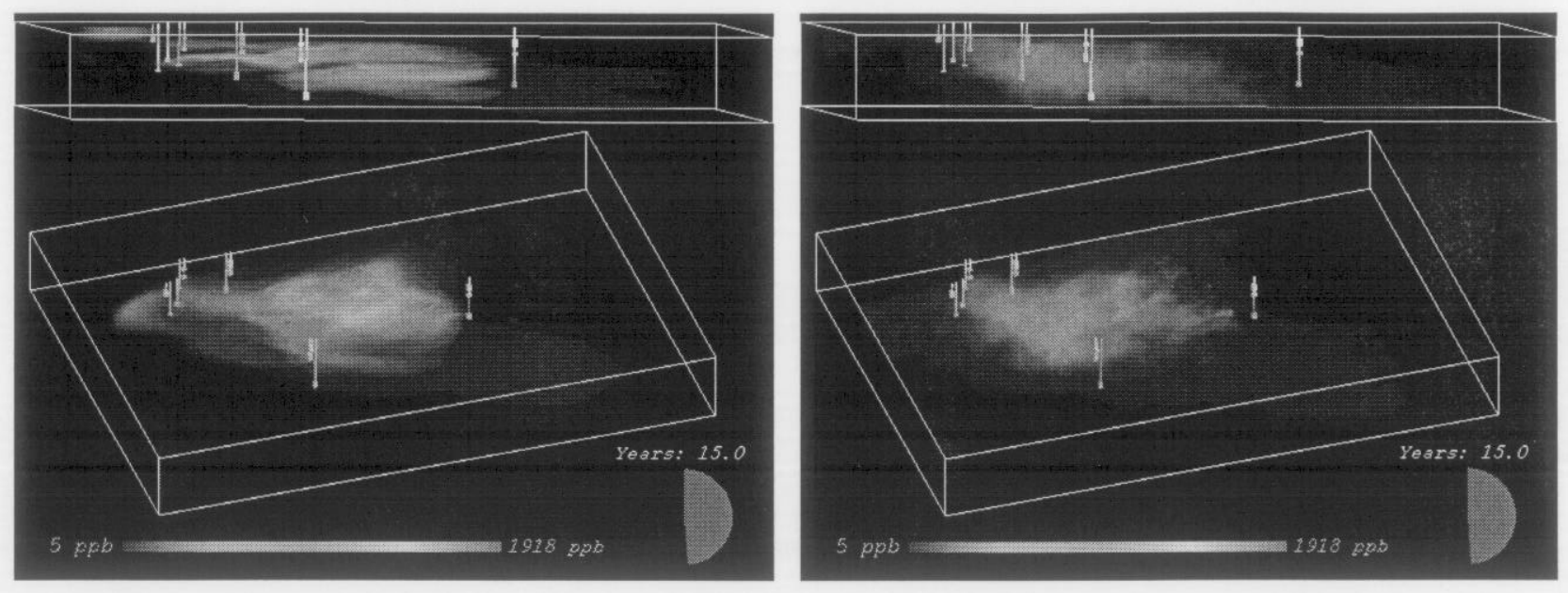

Figure 1: Snap-shot in time of a contaminant migrating through a homogeneous (left) and a heterogeneous (right) subsurface medium.

of preferential flow paths. For groundwater remediation simulations, the need to adequately resolve this heterogeneity (length scales on the order of meters) on sites that typically encompass several square kilometers leads to computational domains with upwards of one billion spatial zones. For water resource management simulations, subsurface heterogeneity also plays an important role. But here, sites typically encompass several hundreds of square kilometers, leading to even larger computational domains. In order to solve such large problems, massively parallel processing (MPP) power is needed.

Although parallel processing may be necessary for detailed simulations, it is not sufficient. One important issue that is often overlooked is the need for scalable numerical algorithms. The algorithms used in most of the current simulation codes for the applications of interest do not scale. That is, the amount of work required to solve the problem grows nonlinearly with the problem size. The use of scalable algorithms can decrease simulation times by several orders of magnitude (see, e.g., [3]), which can mean the difference between a two-day run and a half-hour run on an MPP. Furthermore, codes which take advantage of such algorithms are limited only by the size of the available computing platforms.

The goal of this project was to develop and apply advanced computational methodologies for simulating multiphase flow through three-dimensional heterogeneous porous media. We built on past successes in which we demonstrated the feasibility of using massively parallel computers and sophisticated iterative linear solvers to enable these simulations [17, 2, 4, 5, 13, 14]. In previous efforts, the focus was on single-phase flow and the scalable solution of the pressure equations via a multigrid preconditioned conjugate gradient (MGCG) method. In this project, the focus was on developing a fast accurate advection scheme using a new temporal subcycling technique and on the scalable and efficient solution of the nonlinear Richards' equation used to model two-phase (variably saturated) flow. Our computational technologies were applied to the simulation of subsurface fluid flow and chemical transport in the context of two important applications: water resource management and groundwater remediation. Moreover, we implemented these methodologies on a variety of high performance computing platforms, ranging from workstation clusters to massively parallel computers. 


\section{Modeling Flow in Porous Media}

\subsection{Single-Phase, Incompressible Flow}

Our simulation efforts prior to this project involved a single fluid phase containing several chemical constituents that move passively within that phase. The equations describing single-phase, multicomponent flow through porous media consist of mass conservation

$$
\frac{\partial}{\partial t}\left(\phi \rho c_{i}\right)+\nabla \cdot\left(c_{i} \rho \vec{V}\right)=q_{i}+\nabla \cdot\left(\vec{D} \cdot \nabla c_{i}\right)
$$

where $i$ is a component index, Darcy's law (approximating conservation of momentum)

$$
\vec{V}=-\bar{k} \lambda \cdot(\nabla p-\rho \vec{g})
$$

and phase equilibrium

$$
\sum_{i} c_{i}=1
$$

In the single-phase case, the constraint that the fluid fills the pore volume is trivially satisfied eliminating the need to consider phase saturation. In the mass equation, $c_{i}$ is the mass concentration of fluid component $i, \rho$ is the total fluid density, $\phi$ is the void volume fraction (i.e., porosity), $\bar{D}$ is the hydrodynamic dispersion tensor, and $q_{i}$ is a source term for component $i$. Darcy's law relates fluid velocity $\vec{V}$ to fluid pressure $p$ via medium permeability $\bar{k}$, fluid mobility $\lambda$ (i.e., the quotient of density and the fluid viscosity in the single phase case), and gravitational effects.

To solve the above equations, we use an IMPES (IMplicit Pressure, Explicit Saturation) scheme in which we separate the hyperbolic and elliptic parts of the equations and solve for concentrations and fluid pressure separately. We assume that the fluid is incompressible ( $\rho$ is a constant function of time and spatial position) as well as the medium ( $\phi$ is a constant function of time). By summing the mass conservation equations (1) over components, applying phase equilibrium (3), then substituting Darcy's law, we get the following elliptic equation for pressure

$$
-\nabla \cdot(\vec{k} \lambda \cdot(\nabla p-\rho \vec{g}))=\frac{1}{\rho} \sum_{i} q_{i}=q .
$$

Equation (4) is solved first, then used to construct the fluid velocity field. Finally, the mass conservation equations are integrated by applying an explicit, conservative, finite-difference approximation to $(1)$.

\subsection{Variably Saturated Flow (Richards'Equation)}

Two issues that must be considered in water resource management problems are water table fluctuation and water seepage through the unsaturated zone. If the water table drops too low, the available drinking water is reduced, and the possibility exists for leaving a well stranded. If the water table rises too high, the aquifer may not accept additional recharge and may require that more water be pumped out. In coastal aquifers where fresh water is scarce, treated wastewater (grey water) is placed into seepage ponds or rivers at the ground surface, where it then migrates through the unsaturated zone into the aquifer, using the ground as a secondary filter. Since EPA guidelines require grey water to be in the ground for several years before it can be extracted and used again, it is important to know the travel time from seepage pond to extraction well in order to effectively manage water resources. 
To consider the above effects, we need to model the flow of water through a medium filled with air and water (variably saturated flow). One common model is Richards' equation [12]. Unlike full two-phase models where two coupled equations must be solved for two unknowns (see e.g., [8]), Richards' equation formulates the problem as a single equation in terms of a single unknown. This is achieved by making the assumption that the air phase pressure is passive so that the resulting model does not allow for air phase movement. This assumption is a limitation only for cases of contaminant flow in the air phase and for very dry soils where evaporation is prominent. For the water resource management problems we are considering in this project, the Richards' equation assumption is not a limitation.

Richards' equation can be stated as

$$
\frac{\partial(s \rho \phi)}{\partial t}-\nabla \cdot(\bar{k} \lambda \cdot(\nabla p-\rho g))=q
$$

where $p$ is the water pressure, $s(p)$ is the water phase saturation (the fraction of pore space occupied by water $), \rho(p)$ is the water phase density, $\phi(p)$ is the porosity of the medium, $\bar{k}$ is the permeability of the medium, and $\lambda(p)$ is the mobility of water. Note that both porosity and density are now dependent on pressure, allowing a slight compressibility of water and of the medium. Also dependent on pressure is the mobility which is $k_{r}(p) \rho(p) / \mu$, where $k_{r}$ is the relative permeability (the decrease in ability of water to flow due to the presence of an air phase) and $\mu$ is water viscosity. Both saturation and relative permeability are nonlinear functions of pressure. These curves are commonly given as data points, but closed form expressions exist and can be used as approximations to these curves $[10]$.

To solve Richards' equation, we employ a cell-centered finite difference scheme equivalent to the lowest order mixed finite element method. The mixed finite element method applied to Richards' equation was analyzed by Woodward and Dawson where optimal convergence was shown for strictly variably saturated flow and bounds were derived on the error for other flow regimes [19]. For the time discretization, we apply backward Euler time stepping. This method of discretizing Richards' equation leads to a system of discrete nonlinear equations.

\subsection{General Domains and Other Modeling Capabilities}

It is usually not possible to model regional sites such as those that arise in water resource management applications with a parallelepiped (box-shaped) domain. To address this, we developed a "general domain" capability that we then implemented in PARFLOw. Figure 2 shows the domain (with a cutout of the interior) for a regional site in Orange County, CA. Our new general domain capability allows us to represent this domain in a grid-independent way and automatically map it onto a computational grid at runtime. We developed a scalable algorithm to convert the conceptual model to a grid-dependent model, and we developed a cheap, efficient, scheme based on a variant of the octree data structure to store the grid-dependent form of the conceptual model. We can then easily loop over data both inside, outside, or along the boundaries to do computations such as apply boundary conditions in a discretization routine. This new capability allows us to scale the compulational model up or down by simply modifying a few input parameters.

Several additional capabilities were added to PARFLOW to allow more realistic simulations. These included transient conditions (e.g., seasonal rainfall or variable well pumping rates), more general porosity input, simple chemical reactions (e.g., decay rate for contaminants), and linear retardation. We also added a recirculating well capability for the LLNL studies in EPD. 


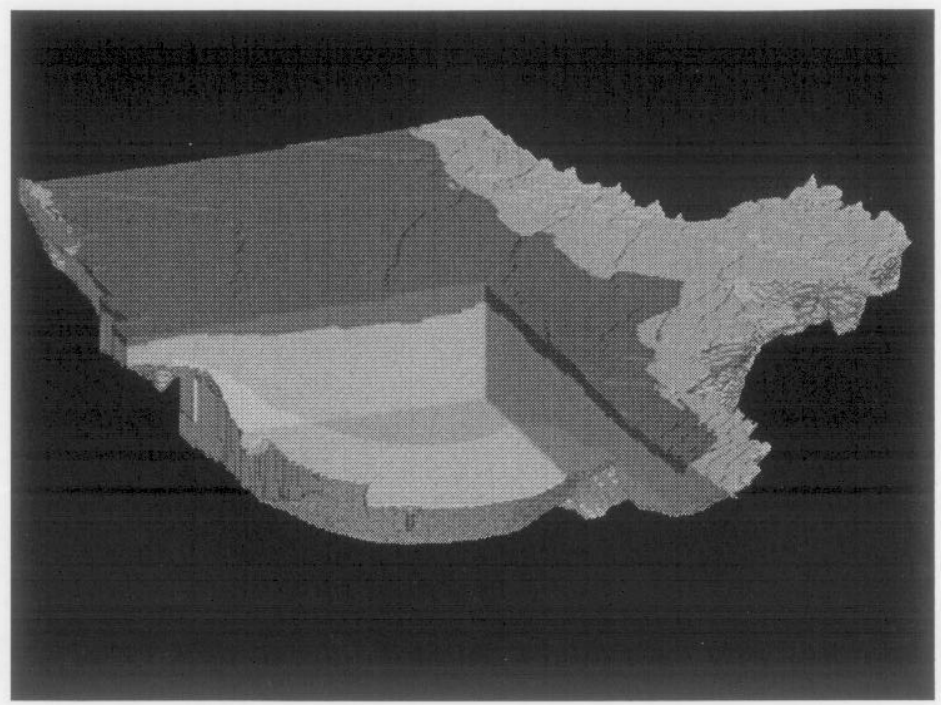

Figure 2: A computational domain for the Orange County regional site.

\section{Computational Technologies}

\subsection{Temporal Subcycling}

To accurately and efficiently solve the mass conservation equation in (1), we developed a novel new time integration technique called temporal subcycling. The goal of temporal subcycling is to employ explicit time integration methods for hyperbolic conservation laws (e.g., Godunov's method) more efficiently by applying the Courant-Friedrichs-Lewy (CFL) stability restriction locally rather than globally. The CFL criterion defines the maximum stable time increment as a function of the maximum characteristic speed in the problem domain. It is often the case in the single-phase flow problems we are considering that large velocity values (typically associated with wells and high conductivity regions) are localized. When these large local velocities are significantly different than those throughout most of the domain, the time increment dictated by the global CFL condition can be too small for most of the computational region. The benefits of temporal subcycling are twofold: (1) time integration becomes more efficient because we reduce the number of timesteps needed throughout much of the domain, and (2) numerical diffusion is reduced by avoiding unnecessarily small time increments in regions where stability considerations allow a much larger timestep to be taken.

The temporal subcycling process consists of two primary phases. The first phase involves the solution of the following constrained minimization problem

$$
\begin{array}{ll}
\operatorname{minimize} & W(\overrightarrow{\mathrm{v}}), \overrightarrow{\mathrm{v}}=\left[\mathrm{v}_{1}, \ldots \mathrm{v}_{m}\right] \in \mathbb{R}^{m} \\
\text { subject to } & \mathbf{A v} \leq \mathbf{b}, \quad \mathbf{A} \in \mathbb{R}^{(m+1) \times m}, \mathbf{b} \in \mathbb{R}^{(m+1)} .
\end{array}
$$

where $W(\overrightarrow{\mathrm{v}})$ is a work function representing the computational effort needed to integrate the entire computational domain over a specified time interval. The unknown, $\vec{v}$, describes a partition of the velocity field. The constraint insures that $\mathrm{v}_{i} \leq \mathrm{v}_{i+1}$ for $i=0, \ldots, m$ where $\mathrm{v}_{0}$ and $\mathrm{v}_{m+1}$ are the minimum and maximum velocity magnitudes, respectively. Note that $m$, the number of refinement levels, is also an unknown. 
The results of the minimization are used to construct a sequence of nested, locally time-refined computational grids that separate the domain into regions where a timestep of similar size is appropriate. During the second phase, the integration process is performed using the sequence of levels in a recursive fashion similar to that which is employed in adaptive mesh refinement (AMR) algorithms [6]. It is important to note that although multiple time scales are regularly used in existing AMR technology, time refinement is usually accompanied by spatial refinement. This has the effect of localizing work, but the amount of local work grows according to the grid refinement used. Furthermore, the primary goals of AMR and temporal subcycling are fundamentally different. In AMR, we wish to minimize the amount of spatial refinement needed to achieve some desired accuracy in the solution. In temporal subcycling, we wish to minimize the amount of temporal refinement needed to retain stability, thereby maximizing computational efficiency.

We developed an algorithm for computing the "optimal" subcycling levels which involves the solution of the constrained minimization problem in (6). To solve this problem, we developed a parallel heuristic to compute the work function, $W(\vec{v})$, and we developed a solver to compute both $\mathrm{v}$ and $m$.

\subsection{Scalable Nonlinear Solvers}

The main algorithmic advancement necessary for modeling variably saturated flow is a robust solver for discrete systems of nonlinear equations. We considered two approaches for developing such a solver. One approach was to solve the nonlinear system directly using a nonlinear multigrid method such as the Full Approximation Scheme (FAS) (see, e.g., [7]), or to use a two-grid discretization scheme such as was done for the nonlinear heat equation by Dawson, Wheeler and Woodward [9]. The other approach was to apply an inexact Newton method to the nonlinear system. In this method, the nonlinear system is solved by an iterative process where each iteration requires the approximate solution of a nonsymmetric Jacobian system.

We investigated the scalability of a new multigrid, preconditioned Newton-Krylov method to solve the nonlinear systems of equations that arise at each timestep. This method uses a globalized, inexact Newton method for linearization, a Krylov iterative method as the linear Jacobian system solver, and the symmetric part of the Jacobian with a multigrid solver as a preconditioner. By using this combination of solvers, we can take advantage of the fast convergence properties of Newton's method, the robustness of a Krylov algorithm, and the parallel scalability of a multigrid preconditioner.

To implement the Newton-Krylov method, we used KINSOL, a package from the parallel variable ordinary differential equation (PVODE) code being developed at LLNL. As a preconditioner for the generalized minimum residual (GMRES) Krylov algorithm, we used the semi-coarsening multigrid method in ParFlow, applied to the symmetric part of the Jacobian. Numerical studies were done to demonstrate the scalability of the method, and we showed scaled speedups in overall solution time of more than $70 \%$, scaling up to 7 million spatial zones on 128 processors of the IBM SP-2 initial delivery system at LLNL [18].

\section{Simulations}

We applied our computational technologies to real problems in water resource management and groundwater remediation applications. The PARFLOW simulator is the primary means by which we did this. We collaborated with colleagues in Environmental Programs (leveraging an LDRD effort there) and with the Orange County Water District to explore the use of our computational methods

in water resource management. Related to this effort, we worked with Brigham Young University 


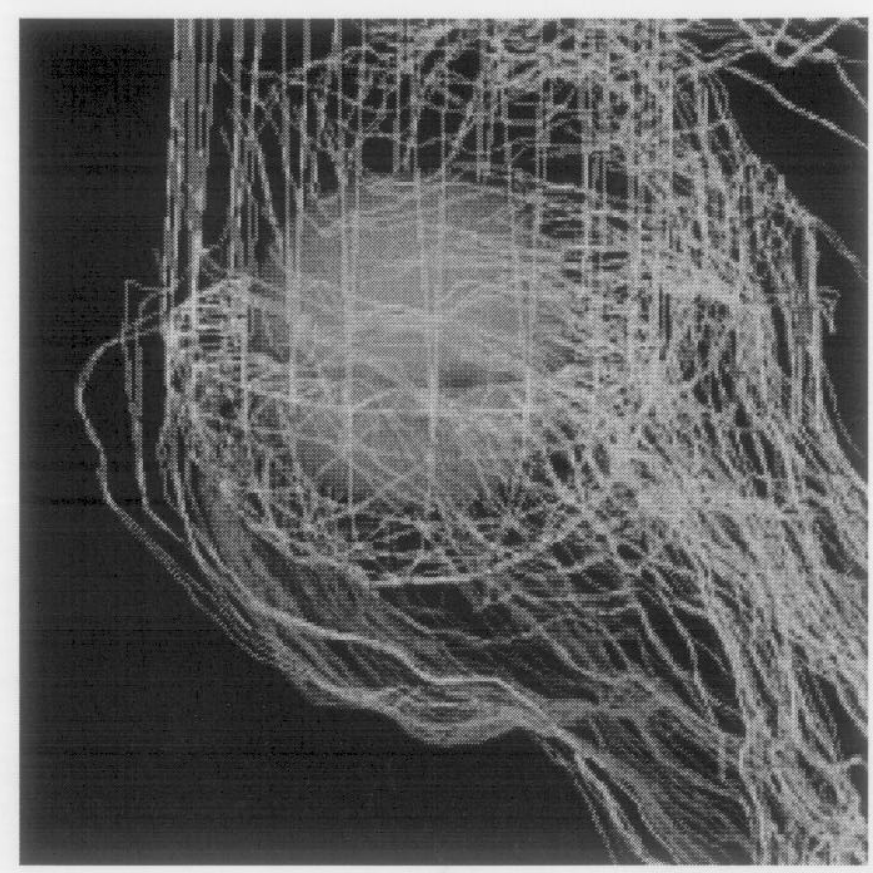

Figure 3: Computed flow field streamlines near the shot point for the Cambric test at the Nevada Test Site. The spherical zone shows the cavity produced by the nuclear test, and the wire frame indicates the disturbed region of the subsurface.

and the Army's Waterways Experiment Station to improve the modeling capabilities of PARFLOW by incorporating it into the Groundwater Modeling System (GMS). Finally, in conjunction with colleagues in Environmental Programs, we used PARFLOW to simulate tritium advection at the Nevada Test Site.

We did preliminary flow calculations for the Orange County water resource management study being done in collaboration with Environmental Programs. Here, significant effort was put into testing the general domain capability for this problem (see Figure 2), and a design was drawn up for the GMS/PARFLOW interface which should considerably simplify the process of defining the conceptual model input for PARFLOW.

We also did simulations on parallelipiped domains to support investigations of aquifer recharge in Orange County. Results suggest that subsurface heterogeneities can have a dramatic effect on the distribution of wastewater age at extraction wells, thereby demonstrating the importance of the affect of geologic heterogeneity on flow paths, mixing, and residence times in the vicinity of recharge basins and wells [15].

We used PARFLOW to do preliminary simulations of tritium advection for the Cambric test at the Nevada Test Site. This shot was chosen because most of the data is unclassified, and there are published simulations of an extensive pumping test near the shot. The results of our first simulation indicate some inconsistencies in the publication on which they were based. Our next simulation was a multi-layered scenario. The breakthrough curves produced by PARFLOW for this case were in good agreement with the measured results. In Figure 3, we show streamlines near the shot for the flow field computed in the second simulation. 


\section{References}

[1] R. Ababou, D. B. Mclaughlin, L. W. Gelhar, and A. F. B. Tompson, Numerical simulation of three-dimensional saturated flow in randomly heterogeneous porous media, Transport in Porous Media, 4 (1989), pp. 549-565.

[2] S. F. Ashby, W. J. Bosl, R. D. Falgout, S. G. Smith, A. F. B. Tompson, and T. J. WILLIAMS, A numerical simulation of groundwater flow and contaminant transport on the CRAY T3D and C90 supercomputers, International Journal of High Performance Computing Applications, 13 (1999), pp. 80-93. Also available as LLNL Technical Report UCRL-JC118635.

[3] S. F. AshBY AND R. D. FALGOUT, A parallel multigrid preconditioned conjugate gradient algorithm for groundwater flow simulations, Nuclear Science and Engineering, 121 (1996), pp. 145-159. Also available as LLNL Technical Report UCRL-JC-122359.

[4] S. F. Ashby, R. D. Falgout, S. G. Smith, And A. F. B. Tompson, The parallel performance of a groundwater flow code on the CRAY T3D, in Proc. Seventh SIAM Conference on Parallel Processing for Scientific Computing, Society for Industrial and Applied Mathematics, 1995, pp. 131-136. Held in San Francisco, February 15-17, 1995. Also available as LLNL technical report UCRL-JC-118604.

[5] S. F. Ashby, R. D. Falgout, and A. F. B. Tompson, A scalable approach to modeling groundwater flow on massively parallel computers, in Proc. U.S. EPA Workshop on Next Generation Environmental Models Computational Methods, SIAM, 1996. Held in Bay City, MI, August 7-9, 1995. To appear. Also available as LLNL technical report UCRL-JC-122591.

[6] M. J. Berger AND P. ColElla, Local adaptive mesh refinement for shock hydrodynamics, J. Comp. Phys., 82 (1989), pp. 64-84.

[7] A. BRANDT, Multigrid techniques: 1984 guide with applications to fluid dynamics, GMDStudien Nr. 85, Gesellschaft für Mathematik und Datenverarbeitung, St. Augustin, 1984.

[8] C. N. Dawson, H. Klie, C. A. San Soucie, And M. F'. Wheeler, A parallel, implicit, cellcentered method for two-phase flow, Texas Inst. for Comp. and Applied Math. 96-35, University of Texas, Austin, TX, Aug. 1996. Submitted to Journal on Computational Geosciences.

[9] C. N. DAWson, M. F. WheEler, AND C. S. WoodWARD, A two-grid finite difference scheme for nonlinear parabolic equations, Center for Applied Scientific Computing UCRL-JC-125599, Lawrence Livermore National Laboratory, Livermore, CA, Oct. 1996. To appear SIAM J. Num. Anal. 1998.

[10] M. T. VAN GENUCHTEN, A closed form equation for predicting the hydraulic conductivity of unsaturated soils, Soil Sci. Soc. Am. J., 44 (1980), pp. 892-898.

[11] National Research Council, Ground Water Models, Scientific and Regulatory Applications, National Academy Press, 1990.

[12] L. A. Richards, Capillary conduction of liquids through porous mediums, Physics, 1 (1931), pp. 318-333. 
[13] A. Tompson, S. Ashby, R. Falgout, And S. Smith, On the role of high performance computing for simulating subsurface flow and chemical migration, in Proc. VIII International Conference on Finite Elements in Fluids-New Trends and Applications, 1994. Held in Barcelona, Spain, September 20-24, 1993. Also available as LLNL Technical Report UCRL-JC-114175.

[14] A. F. B. Tompson, S. F. Ashby, R. D. Falgout, S. G. Smith, T. W. Fogwell, and G. A. LoOSMORE, Use of high performance computing to examine the effectiveness of aquifer remediation, in Proc. X International Conference on Computational Methods in Water Resources, A. Peters, G. Wittum, B. Herrling, U. Meissner, C. Brebbia, W. Gray, and G. Pinder, eds., vol. 2, Kluwer Academic Publishers, Dordrecht, 1994. Held in Heidelberg, Germany, July 19-22, 1994. Also available as LLNL technical report UCRL-JC-115374.

[15] A. F. B. Tompson, S. F. Carle, N. D. Rosenberg, and R. M. Maxwell, Analysis of groundwater migration from artificial recharge in a large urban aquifer: A simulation perspective. Submitted to Water Resources Research. Also available as LLNL technical report UCRL-JC-129563, 1998.

[16] A. F. B. Tompson, R. D. Falgout, S. G. Smith, W. J. Bosl, And S. F. Ashby, Analysis of subsurface contaminant migration and remediation using high performance computing, Advances in Water Resources, 22 (1998), pp. 203-221. Also available as LLNL Technical Report UCRL-JC-124650.

[17] A. F. B. Tompson, N. D. Rosenberg, W. J. Bosl, R. D. Falgout, S. G. Smith, D. E. SHUMAKER, AND S. F. ASHBY, On the use of high-performance simulation in the management of groundwater resources in large aquifer systems, in XXVII IAHR Congress on Water for a Changing Global Community, 1997. Held in San Francisco, CA, August 10-15, 1997. Also available as LLNL technical report UCRL-AR-126359.

[18] C. S. WOODWARD, A neuton-krylov-multigrid solver for variably saturated flow problems, in Proc. of the Twelfth International Conference on Computational Methods in Water Resources, vol. 2, Southampton, 1998, Computational Mechanics Publications, pp. 609 616. Held in Crete, Greece, June, 1998. Also available as LLNL Technical Report UCRL-JC-129371.

[19] C. S. WoOdWARd AND C. N. DAWson, Analysis of expanded mixed finite element methods for a nonlinear parabolic equation modeling flow into variably saturated porous media, Center for Applied Scientific Computation UCRL-JC-125567, Lawrence Livermore National Laboratory, Livermore, CA, Oct. 1996. Submitted to SIAM J. Num. Anal. 